\title{
Decentralized Predictive Control of Multi-Reach Canals
}

\author{
M. Gómez, J. Rodellar, F. Vea, J. Mantecón, J. Cardona \\ School of Civil Engineering, Technical University of Catalonia \\ 08034 Barcelona, Spain
}

\begin{abstract}
ABSTRAC'I
'This paper presents a digital control scheme for water level regulation in canals. The scheme considers a prototype canal composed by a series of pools connected with active gates. A control system is designed for each pool with the objective of manipulating the upstream gate in order to drive and maintain the downstream level at desired values. Fach control system is composed by two controllers: one implements a discrete time predictive control law that gives the desired discharge through the gate as to ensure the downtream desired level; another one implements a local control law to move the gate to achieve the desired discharge. The paper presents the formulation of the global control scheme and shows results from simulations to illustrate and discuss the performance in different operation scenarios.
\end{abstract}

\section{INTRODUCTION}

In the last years it has been growing the interest in introducing automatic control tools to improve the water delivery efficiency in irrigation canals. Different control approaches have boen proposed and reported in the literature. Among them, predictive control has been considered based on the nature of the irrigation control problem and the efficiency shown by predictive controllers in practical applications in a variety of areas. The fundamentals of predictive control invoked in this paper follow the approach of [1]. These fundamentals were already conceptually invoked for irrigation canal control in $[2,3]$, designing stable controllers for on-demand operation of single reaches. In this paper predictive control is used for a multi-reach canal with the objective of level regulation. The regulation scheme has a decentralized setting where the global canal is seen as a set of interconnected reaches designing controllers for each one of them. A centralized nultivariable predictive controller is described in [4]. Several authors have approached the control of irrigation canals via predictive controllers. Significant references are found in $[5-8]$.

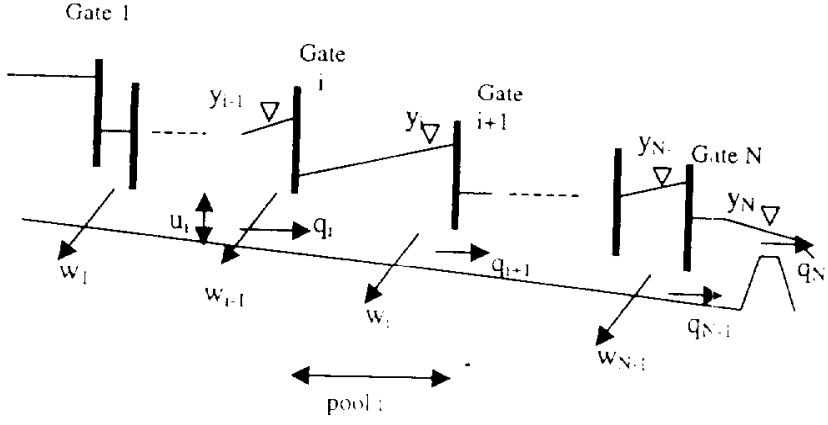

Figure 1: Canal scheme.

\section{OVERALL SYSTEM}

The overall system is a canal with a series of $N$ pools, gates and offtakes as illustrated in Figure 1. The upstream and downstream bounds of the canals are such that there is a reservoir upstream of gate 1 and a weir at the downstream end of the pool $Y$. A lateral offtake is placed immediately upstream of each gate. In this paper, for the sake of simplicity, offtakes are supposed to be weirs, but the procedure presented herein can be readily adapted to other kinds of offtakes.

The overall control scheme is composed by $N$ control systems with the particular objective of manipulating the opening of each gate in order to drive and maintain the setpoint level at the downstream end of the corresponding pool. The objective for the control systern $i$ is to manipulate the opening $w_{i}$ to ensure the level $y_{2}$ to be maintained at the desired setpoint $y_{i}^{c p}$

The design and the implementation of each control system $t$ is undertaken by means of two controllers which will be referred to as downstream controller $i$ and local controller $i$ respectively. The downstream controller $i$ is formulated through a discrete time control algorithm 


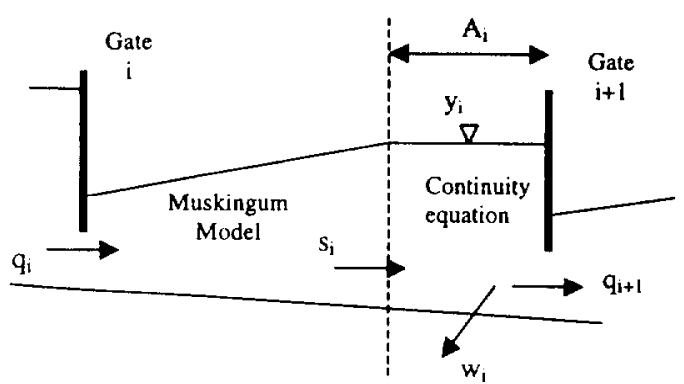

Figure 2: Model of pool $i$.

which, at each sampling instant $k=0,1,2, \ldots$, gives the desired discharge $q_{i}$ through gate $i$ for the next sampling instant $k+1$ such that the downstream level $y_{i}$, as predicted by a model for a future instant, is achieved. The local controller $i$ is designed to move the gate $i$ between instants $k$ and $k+1$ with the purpose of meeting the desired flow at $k+1$ as given by the master controller $i$.

For the design of the downtream controller $i$, this paper relies on the combination of a strategy of predictive control with a simplified model of the pool $i$. The present local controller $i$ is based on a continuous time PID having the gate opening $u_{i}$ as manipulated variable and the discharge $q_{i}$ as controlled variable. The setpoint for this PID controller is changed at each sampling time $k$ according to the desired discharge generated by the predictive downstream controller $i$.

\section{PREDICTIVE DOWNSTREAM CONTROLLER}

Predictive control essentially relies on the use of a model able to predict the system output as a function of the system inputs on a moving horizon scenario, and to compute the control sequence that makes the predicted output to follow a desired trajectory through the minimization of a performance criterion [refs]. The following three subsections present the predictive model adopted in this paper, the control algorithm and the way in which unknown future inputs are estimated for prediction purposes.

\section{Predictive model}

The purpose is to build a simplified model of the pool $i$ involving the discharges $q_{i}$ and $q_{i+1}$ through gates $i$ and $i+1$ respectively, the discharge $w_{i}$ through the offtake $i$ and the downstream water level $y_{1}$. This model describes the pool as composed by a single reach followed by a storage zone, as illustrated in Fig. 2
The single reach is described by a difference equation modcl that relates the upstream and downstream discharges $q_{i}$ and $s_{i}$ respectively in the form

$$
s_{i}(k)=\sum_{j=1}^{n} \nu_{i j}(k) s_{i}(k-j)+\sum_{j=1}^{m} \mu_{i j}(k) q_{\imath}(k-j)
$$

where $k$ denotes the sampling instant $k=0,1,2, \ldots$ Parameters $\nu_{i}(k)$ and $\mu_{i j}(k)$ can be obtained based on system identification approaches or based on physical modelling concepts. They can be obtained off-line and kept constant $\left(\nu_{i j}, \mu_{i j}\right)$ along the operation of the cuntrol system. Or, alternatively, they can be updated on-line as $\nu_{i j}(k)$ and $\mu_{i j}(k)$ in a setting of adaptive control.

By the way of example, we may consider a particularly simple form of model (1) by using the so called Muskingum model

$$
s_{i}(k)=\nu_{i o} s_{i}(k-1)+\mu_{i \circ} q_{2}(k)+\mu_{i 1} q_{3}(k-1)
$$

where the three parameters are given by

$$
\begin{aligned}
\nu_{i o} & =\frac{2 K_{i}\left(1-X_{i}\right)-T}{T+2 K_{i}\left(1-X_{i}\right)}, \quad \mu_{i o}=\frac{T-2 K_{i} X_{i}}{T+2 K_{i}\left(1-X_{i}\right)} \\
\mu_{i 1} & =\frac{T+2 K_{i} X_{i}}{T+2 K_{i}\left(1-X_{i}\right)}
\end{aligned}
$$

where $H_{\mathfrak{t}}$ and $X_{\mathfrak{t}}$ are calibrated hydraulic parameters and $T$ is the sampling period. The storage zone can be modelled (in continuous time) by the differential equation

$$
A_{i} \frac{d y_{i}}{d t}=s_{i}(t)-q_{i+1}(t)-w_{i}(t)
$$

where $A_{i}$ is the section of the storage zone.

By discretizing equation (4) and combining it with model (1) we get

$$
\begin{aligned}
& \Delta y_{i}(k)=\sum_{j=1}^{n} a_{i j} \Delta y_{i}(k-j)+\sum_{j=0}^{m} b_{\imath \jmath} \Delta q_{i}(k-j) \\
& +\sum_{j=0}^{p_{1}} c_{i j} \Delta q_{i+1}(k-j)+\sum_{j=0}^{p_{2}} d_{i j} \Delta w_{i}(k-j)
\end{aligned}
$$

where $\Delta$ denotes the incremental operator which, for a generic variable $y$, gives $\Delta y(k)=y(k)-y(k-1)$. Parameters in (5) are given by appropriate expressions. In the subsequent formulation, these parameters are assumed constant for the sake of simplicity. By using model (5) at each sampling instant $k$, we may predict the incremental 
water level over a prediction interval $[k, k+\lambda]$ in the form

$$
\begin{aligned}
& \Delta \hat{y}_{i}(k+l \mid k)=\sum_{j=1}^{n} a_{i j} \Delta \hat{y}_{i}(k+l-j \mid k) \\
& +\sum_{j=0}^{m} b_{i j} \Delta \hat{q}_{i}(k+l-j \mid k) \sum_{j=0}^{p_{1}} c_{i j} \Delta \hat{q}_{i+1}(k+l-j \mid k) \\
& +\sum_{j=0}^{p_{2}} d_{i j} \Delta \hat{w}_{i}(k+l-j \mid k), \quad(l=1,2, \ldots, \lambda)
\end{aligned}
$$

where $\Delta \hat{y}_{i}(k+l \mid k)$ denotes the sequence of increments of the level $y_{i}$ predicted at instant $k$ for future instants $k+l$ in response to the sequences of incremental inputs $\Delta \hat{q}_{i}, \Delta \hat{q}_{i+1}$ and $\Delta \hat{w}_{i}$ over the interval $[k, k+\lambda]$. This prediction is redefined at each sampling time $k$ in the form

$$
\begin{array}{ll}
\Delta \hat{y}_{i}(k+1-j \mid k) & =\Delta y_{i}(k+1-j), j=1, \ldots, n \\
\Delta \hat{q}_{i}(k+1-j \mid k) & =\Delta q_{i}(k+1-j), j=1, \ldots, m \\
\Delta \hat{q}_{i+1}(k+1-j \mid k) & =\Delta q_{i+1}(k+1-j), j=1, \ldots, p_{1} \\
\Delta \hat{w}_{i}(k+1-j \mid k) & =\Delta w_{i}(k+1-j), j=1, \ldots, p_{2}
\end{array}
$$

Notice that variables without hat accent denote quantitites that are known at time instant $k$. Thus, the redefinition of the predictive model in (7) means that the controller $i$ will need information on the actual level at downstream end $\left(y_{i}\right)$, the discharges through gates $i$ and $i+1\left(q_{i}\right.$ and $q_{i+1}$ respectively) and the discharge through offtake $i\left(w_{i}\right)$ at each sampling time $k$. Also notice that the prediction defined by the model (6) involves future variables on the interval $[k, k+\lambda]: \Delta \hat{q}_{i}(k+l-j \mid k)$ is a control sequence to be determined by the master controller design, while $\Delta \hat{q}_{i+1}(k+l-j \mid k)$ and $\Delta \hat{w}_{i}(k+l-j \mid k)$ are seen as disturbances which cannot be manipulated by the controller. Let us assume in this section that these disturbance sequences are available (in a further subsection we will deal with how to estimate them). Then, by using model (6) we may predict the sequence $\Delta \hat{y}_{i}(k+l \mid k)$ having the control sequence $\Delta \hat{q}_{i}(k+l \mid k)(l=1,2, \ldots, \lambda)$ as unknown.

\section{Predictive controller}

In order to obtain the incremental control sequence $\Delta \hat{q}_{i}(k+l \mid k)(l=1,2, \ldots, \lambda)$, we may impose the minimization of a performance criterion. Different criteria can be used [1], but a particular simple case, with satisfactory results in previous applications $[2,3]$, can be defined by the following two conditions:

$$
\begin{array}{ll}
\hat{y}_{i}(k+\lambda \mid k) & =y_{i}^{T}(k+\lambda \mid k) \\
\Delta \bar{q}_{i}(k+l \mid k) & =0, \quad l=2,3, \ldots, \lambda
\end{array}
$$

This performance criterion has an intuitive basis: we aim to find the control sequence that, being constant between instants $k+1$ and $k+\lambda$, renders the output $y_{i}$ predicted for the last prediction time $k+\lambda$ be equal to a reference value $y_{i}^{r}$. This reference value can be chosen as belonging to a desired trajectory converging to the setpoint $y_{i}^{8 p}$. Imposing a constant control sequence is a practical manner of reducing the number of unknowns to only one, that is $\Delta \hat{q}_{i}(k+1 \mid k)$.

By using the predictive model (6) recursively from the initial conditions ( 7 ), we may write

$$
\begin{aligned}
& \Delta \hat{y}_{i}(k+l \mid k)=\sum_{j=1}^{n} e_{i j}^{(l)} \Delta y_{i}(k+1-j) \\
&+ \sum_{j=1}^{m} g_{i j}^{(l)} \Delta q_{i}(k+1-j)+\sum_{j=1}^{p_{1}} f_{i j}^{(l)} \Delta q_{i+1}(k+1-j) \\
&+ \sum_{j-1}^{p_{2}} h_{i j}^{(l)} \Delta w_{i}(k+1-j)+\sum_{j-1}^{l} g_{i o}^{(l+1-\jmath)} \Delta \hat{q}_{i}(k+j \mid k) \\
&+ \sum_{j=1}^{l} f_{i o}^{(l+1-j)} \Delta \hat{q}_{i+1}(k+j \mid k)+h_{i o}^{(l+1-j)} \Delta \hat{w}_{i}(k+j \mid k) \\
& l=1,2, \ldots, \lambda
\end{aligned}
$$

where the coefficients are obtained from the parameters of the predictive model (6) using the following recursive algorithms:

$$
\begin{aligned}
& e_{i j}^{(l)}=e_{i 1}^{(l-1)} a_{i j}+e_{i, j+1}^{(l-1)} ; j=1, \cdots, n \quad l=2, \cdots, \lambda \\
& g_{i j}^{(l)}=e_{i 1}^{(l-1)} b_{i j}+g_{i, j+1}^{(l-1)} j=0,1, \cdots, m ; l=2, \cdots, \lambda \\
& f_{i j}^{(l)}=e_{i 1}^{(l-1)} c_{i j}+f_{i, j+1}^{(l-1)} j=0,1, \cdots, p_{1} ; l=2, \cdots, \lambda \\
& h_{i j}^{(l)}=e_{i 1}^{(l-1)} d_{i j}+h_{i, j+1}^{(l-1)} j=0,1, \cdots, p_{2} ; l=2, \cdots, \lambda \\
& e_{i j}^{(1)} \quad=a_{i j} \quad j=1, \cdots, n \\
& g_{i j}^{(1)} \quad=b_{i j} \quad j=0,1, \cdots, m \\
& f_{i j}^{(1)}=c_{i j} \quad j=0,1, \cdots, p_{1} \\
& h_{i j}^{(1)} \quad=d_{i j} \quad j=0,1, \cdots, p_{2} \\
& e_{i, n+1}^{(l-1)}=g_{i, m+1}^{(l-1)}=0 \quad j=2, \cdots, \lambda \\
& f_{i, p_{1}+1}^{(l-1)}=h_{i, p_{2}+1}^{(l-1)}=0 \quad j=2, \cdots, \lambda
\end{aligned}
$$

By summing up the $\lambda$ equations of (9) and using (7) and the second condition of (8), the predicted water level for $k+\lambda$ can be written in the form 


$$
\begin{gathered}
\hat{y}_{i}(k+\lambda \mid k)=y_{i}(k)+\sum_{j=1}^{n} \alpha_{i j}^{(\lambda)} \Delta y_{i}(k+1-j) \\
+\sum_{j=1}^{m} \beta_{i j}^{(\lambda)} \Delta q_{i}(k+1-j)+\sum_{j=1}^{p_{1}} \gamma_{i j}^{(\lambda)} \Delta y_{i+1}(k+1-j) \\
+\sum_{j=1}^{p_{2}} \delta_{i j}^{(\lambda)} \Delta w_{i}(k+1-j)+\beta_{i o}^{(\lambda)} \Delta \hat{q}_{i}(k+1 \mid k) \\
+\sum_{j=1}^{\lambda} \gamma_{i o}^{(\lambda+1-j)} \Delta \hat{q}_{i+1}(k+j \mid k)+\delta_{i o}^{(\lambda+1-j)} \Delta \hat{w}_{i}(k+j \mid k)
\end{gathered}
$$

where

$$
\begin{array}{ll}
\alpha_{i j}^{(r)}=\sum_{l=1}^{r} e_{i j}^{(l)}, & \beta_{i j}^{(r)}=\sum_{l=1}^{r} g_{i j}^{(l)} \\
\gamma_{i j}^{(r)}=\sum_{l=1}^{r} f_{i j}^{(l)}, & \delta_{i j}^{(r)}=\sum_{l=1}^{r} h_{i j}^{(l)}
\end{array}
$$

Now, just imposing the first condition of (8) we have

$$
\begin{aligned}
& \Delta \hat{q}_{i}(k+1 \mid k)=\frac{y_{i}^{r}(k+\lambda \mid k)-y_{i}(k)-\hat{c}_{i}(k+1 \mid k)}{\beta_{i o}^{(\lambda)}} \\
& \hat{c}_{i}(k+1 \mid k)= \\
& \sum_{j=1}^{n} \alpha_{i j}^{(\lambda)} \Delta y_{i}(k+1-j)+\sum_{j=1}^{m} \beta_{i j}^{(\lambda)} \Delta q_{i}(k+1-j) \\
& +\sum_{j=1}^{p_{1}} \gamma_{i j}^{(\lambda)} \Delta q_{i+1}(k+1-j)+\sum_{j=1}^{p_{2}} \delta_{i j}^{(\lambda)} \Delta w_{i}(k+1-j) \\
& +\sum_{j=1}^{\lambda} \gamma_{i \omega}^{(\lambda+1-j)} \Delta \hat{q}_{i+1}(k+j \mid k)+\delta_{i o}^{(\lambda+1-j)} \Delta \hat{w}_{i}(k+j \mid k)
\end{aligned}
$$

Finally, the control discharge for instant $k+1$ is

$$
\hat{q}_{i}(k+1 \mid k)=\Delta \hat{q}_{i}(k+1 \mid k)-q_{i}(k)
$$

Nutice that, in order to compute, at each sampling instant $k$, the desired discharge $\hat{q}_{i}(k+1 \mid k)$, we need to know the past and present values of the downstream level $y_{i}$, the discharges at gates $i$ and $i+1$ and the discharges at the offtake $i$. We also require the future values of the discharges at gate $i+1$ and offtake $i$, that is $\Delta \hat{q}_{i+1}(k+\jmath \mid k)$ and $\Delta \hat{w}_{i}(k+j \mid k)$ respectively, over the prediction horizon $j=1, \ldots, \lambda$. Present values are known through measurement sensors. Future values have to be estimated. The next subsection describes one way to perform this estimation.

\section{Estimation of future discharges}

The control law formulated in the previous subsection is based on a model predicting the pool behaviour over a time horizon $[k, k+\lambda]$. We remark that, while $k$ denotes the real sampling instant in which the control cperation is performed, the future time instants denoted as $k+$ $1|k, \ldots, k+\lambda| k$ represent a fictitious time sequence. In order to estimate the discharges $\dot{q}_{i+1}(k+j \mid k)$ and $\tilde{w}_{\mathbf{z}}(k+$ $j \mid k)$ at these fictitious time instants, we will assume that, at the final instant $k+\lambda$, each controlled pool $i$ is in a steady state in which the downstream level has reached its prescribed setpoint $y_{i}^{s p}$.

Let us consider the pool $N$ in the scheme of Figure 1. We will consider that the steady state hydraulic relation between the offtake discharge $w_{N}$ and the water level $y_{N}$ is known and modelled by a function $f_{N}$ that characterizes the specific offtake structure. According to the steady statie assumption at the final instant $k+\lambda \mid k$, we consider $\hat{w}_{N}(k+\lambda \mid k)=f_{N}\left(y_{N}^{s p}\right)$. For instants $k+1|k, \ldots, k+\lambda-1| k$ we assume a linear variation from the actual value at instant $k, w_{N}(k)$, to the fiulal value that is,

$$
\begin{aligned}
& \Delta \hat{w}_{N}(k+j \mid k)=\frac{\hat{w}_{N}(k+\lambda \mid k)-w_{N}(k)}{\lambda} \\
& \Delta \hat{w}_{N}(k+\lambda+1 \mid k)=0 ; \quad j=1, \ldots, \lambda
\end{aligned}
$$

A similar assumption can be made for the discharge $\hat{q}_{N+1}$ at the downstream end of the canal scheme, so that $\Delta \hat{q}_{N+1}(k+j \mid k)$ can be estimated for $j=1,2, \ldots, \lambda+1$ as in (17). Let us now consider a generic intermediate pool $i$. Proceeding as for the pool $N$, we assume $\hat{w}_{\imath}(k+\lambda \mid k)=f_{\imath}\left(y_{i}^{s p}\right)$ and then write

$$
\begin{aligned}
& \Delta \hat{w}_{i}(k+j \mid k)=\frac{\hat{w}_{\imath}(k+\lambda \mid k)-w_{\imath}(k)}{\lambda} \\
& \Delta \hat{w}_{i}(k+\lambda+1 \mid k)=0 ; \quad{ }_{j}=1, \ldots, \lambda
\end{aligned}
$$

For the downstream discharge we consider continuity:

$$
\begin{aligned}
\hat{q}_{i+1}(k+\lambda \mid k) & =\hat{w}_{i+1}(k+\lambda \mid k) \ldots+\hat{w}_{N}(k-\lambda \mid k) \\
& +\hat{q}_{N+1}(k+\lambda \mid k)
\end{aligned}
$$

to estimate the steady state downstream discharge at instant $k+\lambda$. For instants $k+1|k, \ldots, k+\lambda| k$ we again consider a linear variation from the actual value at instant $k, q_{2+1}(k)$, to the one given by (19):

$$
\begin{array}{ll}
\Delta \hat{q}_{i+1}(k+j \mid k) & =\frac{\hat{q}_{i+1}(k+\lambda \mid k)-q_{i+1}(k)}{\lambda} \\
\Delta q_{i+1}(k+\lambda+1 \mid k) & =0 ; \quad j=1,2, \ldots, \lambda
\end{array}
$$

In summary, starting from pool $N$, and going backwards to pool 1, we may end up with the estimation of the discharges $\Delta \hat{w}_{i}(k+j \mid k)$ and $\Delta \hat{q}_{i+1}(k+j \mid k)$ for $j=1, \ldots, \lambda$ for each predictive controller $i=1, \ldots, N$, so that the desired control discharge $\hat{q}_{i}(k+1 \mid k)$ can be computed at each sampling instant $k$ by using (16). Notice that 


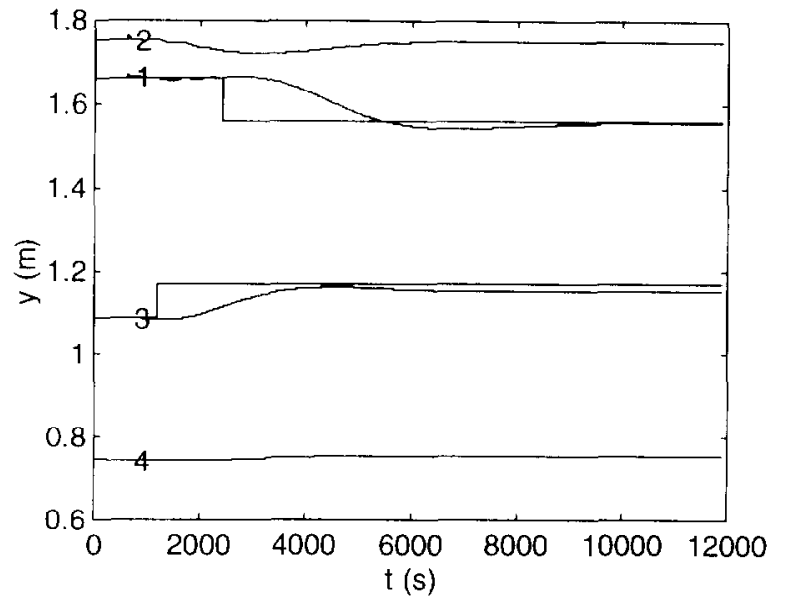

Figure 3: Water levels for setpoint variations.

in order to implement this procedure, each predictive controller $i$ requires, as feedback information, to know the actual discharge through the subsequent gate $i+1$, $q_{i+1}(k)$, the discharge through the oftake $i, w_{i}(k)$, as well as the downstream level $y_{i}(k)$ at instant $k$. It also needs to know the setpoints for each control $i+1, \ldots, N$ downstream of gate $i$.

\section{ILLUSTRATIVE TEST EXAMPLES}

The case study we have selected is a canal composed by 4 reaches (pools). All the reaches are equal, with a length of $1500 \mathrm{~m}$, rectangular cross scction of $2.5 \mathrm{~m}$ width, slope of 0.0005 and Manning roughness coefficient of 0.014. Initial conditions for the system are the following: discharges passing under each gate are $4.27 \mathrm{~m}^{3} / \mathrm{s}$, $3.83 \mathrm{~m}^{3} / \mathrm{s}, 2.5 \mathrm{~m}^{3} / \mathrm{s}$ and $2.5 \mathrm{~m}^{3} / \mathrm{s}$. There is a lateral weir of $1.10 \mathrm{~m}$ at the end of each pool. Water levels downstream each pool are 1.66, 1.75, 1.08 and $0.74 \mathrm{~m}$ respectively.

Downstream of each gate, there is an offtake whose water level is the control objective. The offtake discharge can be produced with a fixed lateral weir or a lateral gate and the purpose of the system is to maintain a desired water level (setpoint). Different setpoint variations have been imposed to assess the ability of the control system, formulated in Section 3 when using the model given by (2), to drive the canal to the desired final state.

As a first example, we have assumed two different variations of the water level setpoints at the end of reaches 1 and 3. Setpoint 1 decreases from $1.66 \mathrm{~m}$ to $1.56 \mathrm{~m}$,

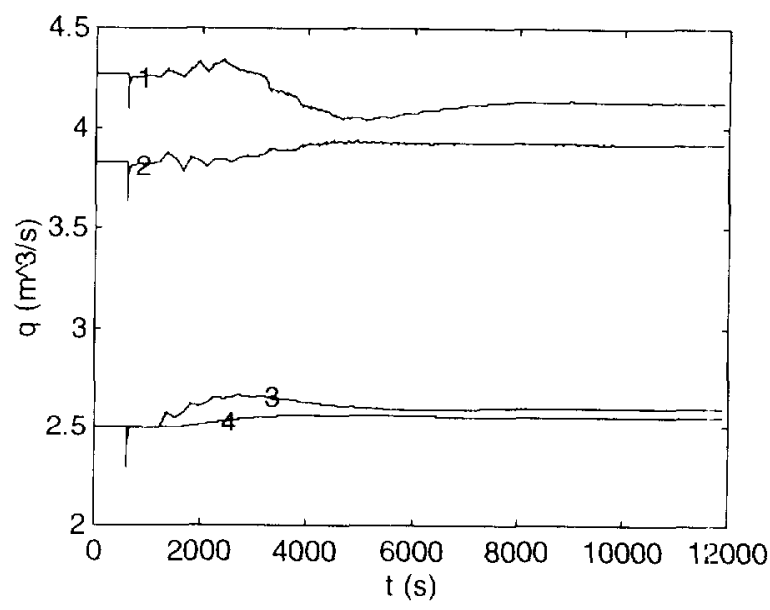

Figure 4: Discharges for setpoint variations.

and setpoint 3 increases from $1.08 \mathrm{~m}$. to $1.18 \mathrm{~m}$. Figure 3 shows the water level evulution, where levels 1 and 3 reach the desired final values. Although water level 2 has no setpoint variation, the movements of gate 3 modify its value and the controller reacts in order to keep the water level. Discharges passing under each gate are shown in Figure 4.

To test the ability of the controller to react in front of unknown perturbations, we have added a consiant discharge of $0.5 \mathrm{~m}^{3} / \mathrm{s}$ at $500 \mathrm{~m}$ downstream of gate 1 . This information is not supplied to the controller, and it only gets knowledge of the perturbation when the water level setpoint is modified due to the incoming discharge. In Figure 5 we can observe how the water level of pool 1 increases and the controller counteracts the effect, driving the water level to its initial value. Discharge passing under gate 1 is reduced to counteract the effect of the perturbation discharge as it can be observed in I'igure 6 .

The situations described above have been numerically simulated replacing the controlled canal by a computational scheme based on the full Saint-Venant equations. The method of characteristics has been used in the numerical solution, considering an explicit approach (space step $20 \mathrm{~m}$ ). The purpose of the simulation has been to assess the effectiveness of the control system in a variety of realistic operating conditions. Thus we have checked if the control system is able to achieve different changes in the water level as well as to react in front of the negative effect of disturbances. The tests can be seen as usual changes in an on-demand water distribution systrm from a steady state to different desired water level. 


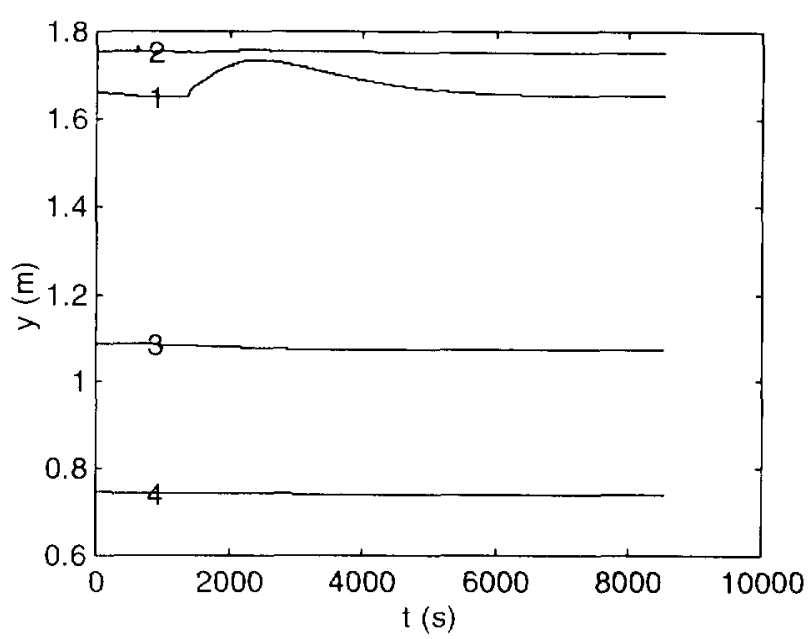

Figure 5: Water levels in front of unknown discharge perturbation.

The whole code, both the controllers and the hydraulic model, has been written as a set of Matlab functions (version 4.2c.1), which are called from the Simulink simulation environment. Thus we can modify both the controller and the hydraulic parameters of the canal in a flexjble interactive manner. The scheme can be seen as a platform for development and testing of different control algorithms.

\section{CONCLUSIONS}

A decentralized control scheme has been proposed for irrigation canals made of a series of pools connected by gates. A predictive controller has been designed to control each pool with the objective of ensuring the setpoint water level at the checkpoint located downstream of the pool. Each control unit works independently and it uses the downstream water level at every reach, and the discharge passing through the control gate as well as the desired downstream discharge as the only feedback information. A simulation scheme has been set up in Matlab allowing easy, flexible design of the controllers and their evaluation

\section{REFERENCES}

[1] J.M. Martín Sánchez, J. Rodellar, Adaptive Predictive Control, Prentice Hall, 1996.

[2] J. Rodellar, M. Gómez, J.P. Martín Vide, "Stable Predictive Control of Open Channel Flow", ASCE J. Irrigation and Drainage Engr., Vol. 115(4), 1989, pp. 701-713.

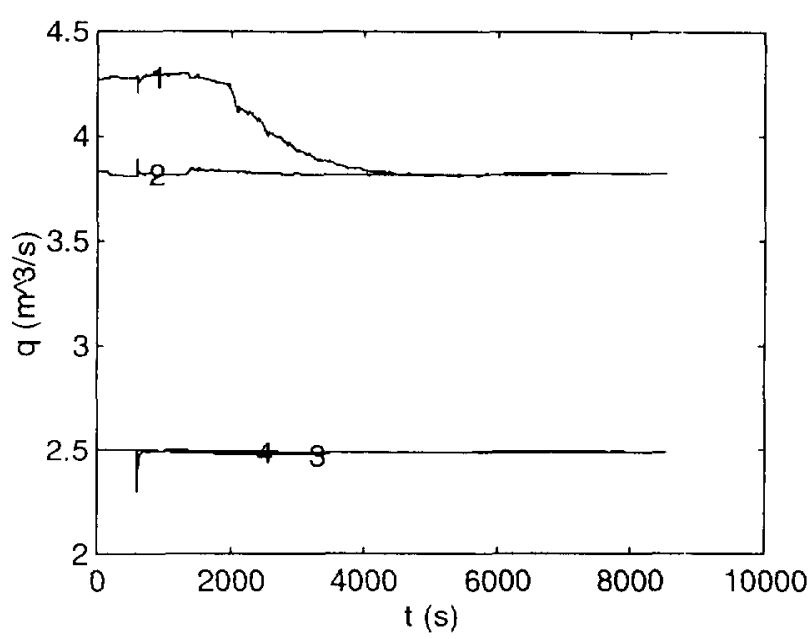

Figure 6: Discharges in front of unknown discharge perturbation.

[3] J. Rodellar, M. Gómez, L. Bonet, "Control Method for On-demand Opcration of Open Channel Flow", ASCE $J$. Irrigation and Drainage Engr., Vol. 119(2), 1993, pp. 225-241.

[4] P.O Malaterre, J. Rodellar, "Multivariable Predictive Control of Irrigation Canals. Design and Evaluation on a 2-Pool Model", Proc. RIC97, Marrakech, Morocco, April 1997, pp. 230-238.

[5] K. Akouz, A. Benhammou, P.O. Malaterre, B. Dahhou, "Predictive Control of an Irrigation Channel", Proc. RIC97, Marrakech, Morocco, April 1997, pp. 209-2211.

[6] J. Cardona, M. Gómez, J. Rodellar, "A Decentralized Adaptive Predictive Controller for Irrigation Canals", Proc. RIC97, Marrakech, Morocco, April 1997, pp. 215219.

[7] J.M. Compas, J.C. Pagès, "Regulation by F'redictive Control and Embedded Knowledge Based Modes", Proc. RIC97, Marrakech, Morocco, April 1997, pp. 220-2229.

[8] S. Sawadogo, P.O. Malaterre, A. Niang, R. Faye, "Multivariable Generalized Predictive Control with Feedforward for On-demand Operation of Irrigation Canals", Proc. RIC97, Marrakech, Morocco, April 1997, pp. 249257.

\section{Acknowledgements}

This work is supported by CICYT (Water Resources Program) under project HID96-1417. 